\title{
Psychiatric Disorder in a Patient with Pituitary Tumor
}

\author{
Rahman MM $^{1}$, Khan SIMKN ${ }^{2}$, Khan RA $^{3}$, Islam MR $^{4}$, Sarker $\mathrm{MH}^{5}$, Huque $\mathbf{M}^{6}$, Ahsan $\mathrm{MN}^{7}$
}

Conflict of interest: There is no conflict of interest relevant to this paper to disclose.

Funding Agency: Was not funded by any institute or any group.

Contribution of Authors: Rahman MM was Principal investigator, Khan SIMKN help for protocol preparation, Khan RA, Islam MR, Sarker MH, Huque M help for data collection and hsan MN help for editorial formatting

Copyright: @2019 Bang. JNS published by BSNS. This article is published under the creative commons CC-BY-NC license.

This license permits use distribution (https://creativecommons.orgf/ licences/by-nc/4-0/) reproduction in any medium, provided the original work is properly cited, and is not used for commercial purposes.

Received: 30 February, 2019

Accepted: 14 March, 2019

\begin{abstract}
We report a case of a patient with pituitary tumor with psychiatric disorder, which posed a significant diagnostic dilemma and management challenge for the treating teams of neurosurgery, endocrinology and psychiatry. A more comprehensive endocrine work-up of atypical presentations can be considered in some cases. The criteria for determining when endocrine work should be considered should be investigated further.
\end{abstract}

Keywords: Psychiatric disorder, Pituitary tumor, Endocrinology

Bang. J Neurosurgery 2019; 9(1): 75-77

\section{Introduction:}

A 65-year - old male patient presented to the Emergency department as anxiety, depression and apathy and was sent to medicine department for evaluation and they found hypopituitarism and then endocrine department was asked for opinion and they advised for MRI which revealed Pituitary Macroadenoma and sent to the Neurosurgery Department for opinion. During his admission, the clinical condition of the patient deteriorated and led to a sudden altered mental status that led to a transfer to the medical floor and further investigation. The laboratory studies revealed hyponatremia and a grossly low hormone profile. The progression of these events casts doubt on our diagnosis as the primary cause of the symptoms of the patient. The clinical condition of the patient only improved when his endocrinopathy, in addition to antipsychotics, was treated with hormone replacement, fluids and electrolyte correction. We revised the diagnosis of neuropsychiatric manifestations secondary to panhypopituitarism due to pituitary macroadenoma.

\section{Case Presentation:}

The patient could not give an account of the admission circumstances. He appeared disheveled, his thinking process was non - linear, illogical and lacked associational quality with a grossly disorganized speech and behavior. His content of thought was important for illusions of paranoia, grandiosity and religious themes. Initial routine laboratory research was within normal limits and the urine toxicology of illicit drugs was negative. The patient was determined to be acutely psychotic, dangerous for him and others and in need of stabilization.

1. Dr. Md. Moshiur Rahman, Assistant Professor (CC) Neurosurgery Department, Holy Family Red Crescent Medical College

2. S.I.M. Khairun Nabi Khan, Assistant Professor, Neurosurgery Department, BSMMU

3. Dr. Robert Ahmed Khan, Medical Officer, Neurosurgery Department, BSMMU

4. Dr Md Rokibul Islam, Medical Officer, Neurosurgery Department, BSMMU

5. Prof. Mainul Haque Sarker, Ex- Professor and Head, Neurosurgery Department, Dhaka Medical College

6. Dr Mahmudul Huque, Associate Professor and Head, Endocrinology Department, Holy Family Red Crescent Medical College

7. Prof. Dr. Md Nazmul Ahsan, Professor and Head, Department of Psychiatry, Holy Family Red Crescent Medical College

Address of Correspondence: Dr. Md. Moshiur Rahman, Assistant Professor, Neurosurgery Department, Holy Family Red Crescent, Medical College 


\section{Hospital Course:}

After a week of admission, his clinical condition seemed to get worse; he also developed a sudden onset of altered mental status with marked disorientation, increased awareness and worsened visual and additive hallucinations. Further work, including laboratory research, revealed substantial hyponatremia that could have contributed to the marked disorientation. The medical team has been consulted and recommended to be transferred to the medical floor.

Laboratory Investigations: The total blood count (CBC), kidney, liver function tests and urinary toxicology of the patient were within normal limits. Serum sodium and potassium respectively have been $128 \mathrm{mmol} / \mathrm{L}$ (136-144.0mmol / L) and 4.2mmol / L (3.6-5.1). Other routine urine analysis and coagulation profiles, such as the routine chest radiograph and ECG, were also within normal limits. A complete hormonal profile with a clinical suspicion of hypopituitarism was performed when transferred to the medical floors. Cortisol serum (AM) was $1.8 \mathrm{mg} / \mathrm{dL}$ (6.2-19.4mg / dL). Basal IGF1 serum levels were $46 \mathrm{mg} / \mathrm{mL}$ (47-192 mg / mL). In general, the pituitary hormone profile of the patient suggested panhypopituitarianism.

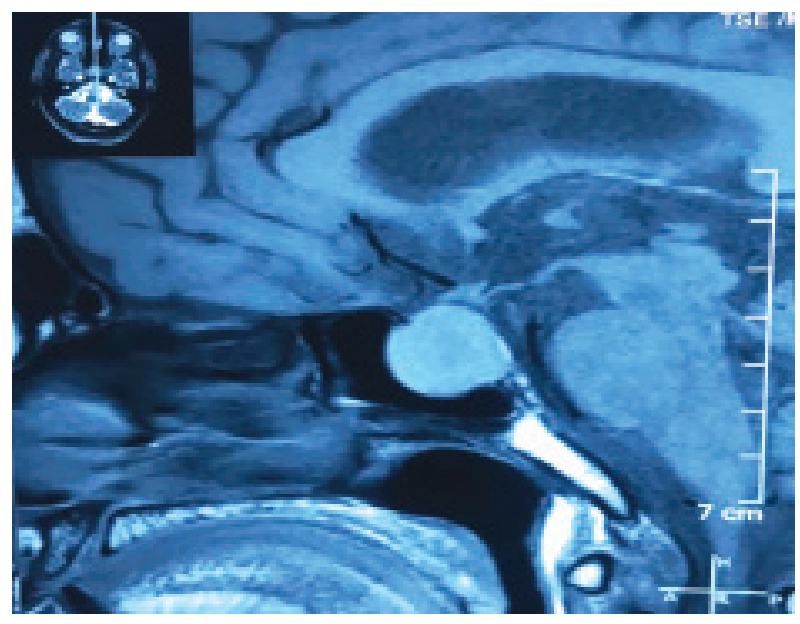

Fig.-1: Pituitary Macroadenoma

Radiologic Investigations: MRI brain imaging with gadolinium injection showed a $2 \mathrm{~cm} \times 2 \mathrm{~cm}$ lesion within the sellaturcica which uptakes the contrast homogenously. During the next few weeks, the patient improved significantly on antipsychotics in the medical floors, Sodium replacement (sodium chloride tabs 1 $\mathrm{g}$ three times daily for hyponatremia) and hormone replacement therapy including $60 \mathrm{mg}$ IVPB (intravenous) hydrocortisone every six hours, $125 \mathrm{mcg}$ daily levothyroxine, Testosterone $200 \mathrm{mg} \mathrm{IM}$ (intramuscular) daily and antipsychotic medication continuation, and risperidone $2 \mathrm{mg} \mathrm{PO}$ (oral) twice daily. He was also placed daily for seizure prophylaxis on tablet phenobarbital $60 \mathrm{mg}$ PO (oral). The mental status of the patient improved significantly and was medically cleared to return to the psychiatric floors.

Then in a private clinic in Dhaka (Comfort Hospital) endoscopic bi-nostril transsphenoidal removal of pituitary tumor was done. The postoperative period was uneventful except the transient diabetes insipidus which was resolved within few days. The patient kept on improving clinically. Antipsychotic drugs were gradually withdrawn. The treating team determined at the discharge that the patient was no longer a danger to himself or to others and that his thinking process was aimed, logical, linear and of good associational quality.

\section{Discussion:}

The pathophysiological presentation of panhypopituitarianism is well documented in the literature, but its possible associated neuropsychiatric manifestations appear to be lacking in data. Cases described in the literature include psychotic presentations from various etiologies, including an ectopic posterior pituitary, Russell's Viper Bite, Sheehan's syndrome, Traumatic Brain Injury (TBI) and glucocorticoid therapy. Mechanisms for psychosis pathogenesis in hypopituitarianism may be caused by interactions between pituitary hormones and the dominant neurotransmitters: Serotonin, dopamine, GABA and glutamate and complex changes in the central nervous system of the metabolism and electrolyte resulting from a combination of hypothyroidism, hypoglycemia and low cortisol. Krishnamurthy et al. (2013) described differentially expressed molecules of the hypothalamic-pituitary adrenal axis such as cortisol in a study of molecular changes in post - mortem pituitary glands from schizophrenia subjects, proadrenocorticotropic hormone, arginine vasopressin precursor, protein associated with agouti, growth hormone, prolactin and secretagogin as well as lipid transport and metabolism related molecules such as A1, A2, C3 and $\mathrm{H}$. Pariante et al., 2014, reported a $10 \%$ larger pituitary volume in patients with HPA axis hyperactivity suggestive first episode psychosis and a $17 \%$ smaller pituitary in patients with established schizophrenia, possibly due to repeated episodes of hyperactivity of the HPA axis. The deregulation of thyroid hormones has also been reported as a common feature in schizophrenia; evidence available suggests that the pituitarythyroid 
axis is involved in serotonergic, dopaminergic, glutamatergic and GABAergic networks, as well as myelinization and inflammation.

The etiology of psychosis in our patient is difficult to determine for two main reasons: firstly, our inability to obtain a comprehensive psychiatric and medical history (which he denies), and secondly, the relatively late onset of psychosis and its history of being free of symptoms for so many years after the pituitary resection. These factors make it problematic to exclude a underlying psychotic disorder; however, it is of clinical significance that once we have added hormone supplementation to the medication regime of the patient, remarkable progress has been achieved.

\section{Conclusion:}

As more reports show the neuropsychiatric manifestations of panhypopituitarism, perhaps a more comprehensive endocrine work-up of atypical presentations can be considered in some cases. The criteria for determining when endocrine work should be considered should be investigated further. The study of such criteria will provide an interesting additional research direction.

\section{References:}

1. J. Alexander, P. Mann Mah, N. Laddipeerla, and T. Mohan, "Panhypopituitarism and psychosis in a male patient," Australianand New Zealand Journal of Psychiatry, vol. 44, no. 4, pp. 393-394, 2010.
2. M. A. Baharom,M. F. I. L. Abdullah, andS.Wahab, "Panhypopituitarismand psychosis: issues and challenges inmanagement," International Medical Journal, vol. 23, no. 2, pp. 132-133, 2016.

3. B. Ratnakaran, V.P.Punnoose,S.Das, andA.Kartha, "Psychosis in secondary empty sella syndrome following a Russell's viper bite," Indian Journal of PsychologicalMedicine, vol. 38, no. 3, pp. 254-256, 2016.

4. B. Tikir, E. Goka, M. C. Aydemir, and S. Gurkan, "Psychotic disorder and sheehanssyndrome: etiology or comorbidity? A case report," Turkish Journal of Psychiatry, vol. 26, no. 2, 2015.

5. Y.-C. Chang, J.-C.Tsai, and F.-Y. Tseng, "Neuropsychiatric disturbances and hypopituitarism after traumatic brain injury in an elderlyman," Journal of the FormosanMedical Association, vol. 105, no. 2, pp. 172-176, 2006.

6. S. I. Hong, D. H. Cho, H. C. Kang, D. J. Chung, and M. Y. Chung, "Acute onset of steroid psychosis with very low dose of prednisolone in Sheehan's syndrome," Endocrine Journal, vol. 53, no. 2, pp. 255-258, 2006.

7. D. Krishnamurthy, L. W. Harris, Y. Levin et al., "Metabolic, hormonal and stress relatedmolecular changes in postmortem pituitary glands from schizophrenia subjects," The World Journalof Biological Psychiatry, vol. 14, no. 7, pp. 478-489, 2013.

8. C. M. Pariante,K. Vassilopoulou,D.Velakoulis et al., "Pituitary volume in psychosis," The British Journal of Psychiatry, vol. 185, no. 1, pp. 5-10, 2004.

9. N. C. Santos, P. Costa, D. Ruano et al., "Revisiting thyroid hormones in schizophrenia," Journal ofThyroid Research, vol. 2012, Article ID 569147, 15 pages, 2012. 